\title{
Predictability of extreme values in geophysical models
}

\author{
A. E. Sterk ${ }^{1}$, M. P. Holland ${ }^{1}$, P. Rabassa ${ }^{2}$, H. W. Broer ${ }^{2}$, and R. Vitolo ${ }^{1}$ \\ ${ }^{1}$ College of Engineering, Mathematics and Physical Sciences, Harrison Building, Streatham Campus, University of Exeter, \\ North Park Road, Exeter, EX4 4QF, UK \\ ${ }^{2}$ Johann Bernoulli Institute for Mathematics and Computer Science, University of Groningen, P.O. Box 407, 9700 AK \\ Groningen, The Netherlands
}

Correspondence to: A. E. Sterk (a.e.sterk@gmail.com)

Received: 10 June 2012 - Accepted: 27 August 2012 - Published: 17 September 2012

\begin{abstract}
Extreme value theory in deterministic systems is concerned with unlikely large (or small) values of an observable evaluated along evolutions of the system. In this paper we study the finite-time predictability of extreme values, such as convection, energy, and wind speeds, in three geophysical models. We study whether finite-time Lyapunov exponents are larger or smaller for initial conditions leading to extremes. General statements on whether extreme values are better or less predictable are not possible: the predictability of extreme values depends on the observable, the attractor of the system, and the prediction lead time.
\end{abstract}

\section{Introduction}

Extreme value theory (EVT) for time series $\left\{X_{i}\right\}_{i=1}^{\infty}$ studies the limiting distribution of the partial maxima $\max \left(X_{1}, \ldots, X_{n}\right)$ as $n \rightarrow \infty$. EVT was originally developed for time series of near-independent random variables (Leadbetter et al., 1983; Coles, 2001; Beirlant et al., 2004), but in the last decade EVT has been extended to chaotic deterministic dynamical systems (Haiman, 2003; Freitas and Freitas, 2008; Freitas et al., 2010; Gupta, 2010; Holland et al., 2012a,b; Felici et al., 2007a,b). In the latter context one considers time series generated by evaluating a scalar observable along evolutions of the system.

In this paper we study the predictability of extreme values, rather than their probability distribution. Lyapunov exponents measure predictability, but they are not very useful in forecasting applications. Firstly, Oseledec (1968) proved that Lyapunov exponents are almost everywhere constant functions of the initial condition. This means that all forecast outcomes are equally (un)predictable regardless of the current state of the system. Secondly, forecasts are typically made ahead for only a few days up to weeks, whereas Lyapunov exponents are asymptotic quantities, which are computed for time tending toward infinity.

Finite-time Lyapunov exponents (FTLEs) measure the exponential growth rate of nearby trajectories over a finite time, and typically they strongly depend on the initial condition. Nese (1989) and Abarbanel et al. (1991) showed that FTLEs for dynamical systems such as the Lorenz-63 model and the Hénon map can be very different along various parts of the attractor. Prasad and Ramaswamy (1999) and Datta and Ramaswamy (2003) demonstrated that distributions of FTLEs in intermittent dynamical systems are non-Gaussian, asymmetric, and often have fat tails. Similarly, Lai (2007) found universal distributions for FTLEs in systems having an attractor consisting of two distinct components. For studies on how FTLEs converge to their infinite-time counterparts see Bailey et al. (1997), Ziehmann et al. (1999, 2000), and references therein.

In this paper we study whether initial conditions leading to extreme values have larger or smaller FTLEs and how this depends on the prediction lead time and the attractor of the system. Section 2 introduces our concept of FTLEs based on norms that are tailored to the observable of interest, such as energy, convection, or wind speed. In Sect. 3 we study the distribution of FTLEs as a function of lead time and threshold for three geophysical models: a spectral truncation of the barotropic vorticity equation and the Lorenz-63 and Lorenz96 models. Section 4 concludes the paper with a discussion. 


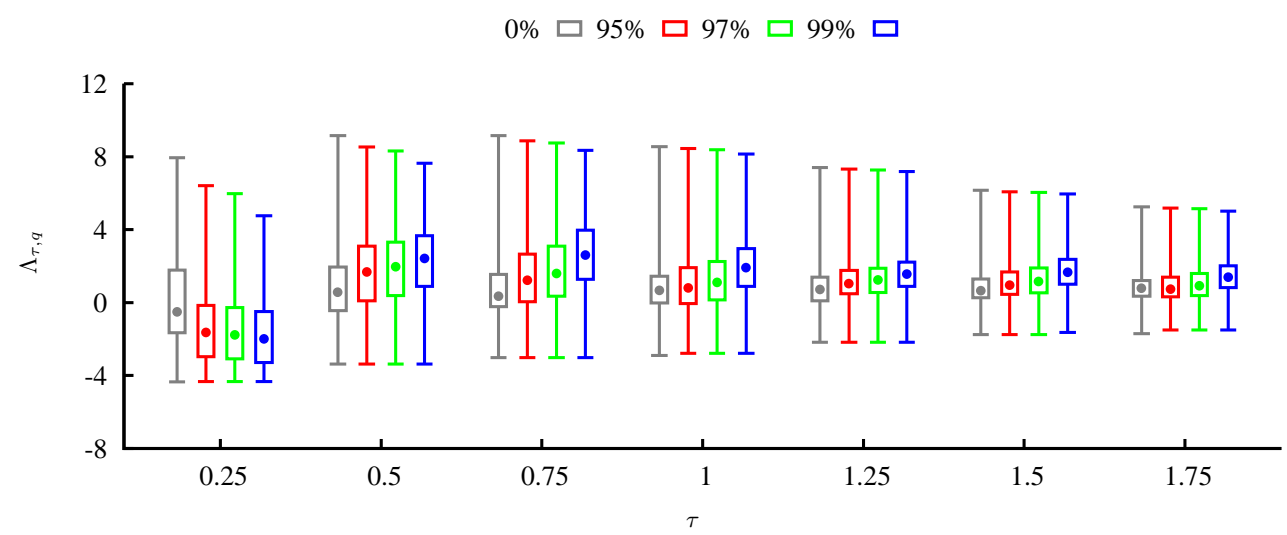

Fig. 1. Distributions of the set $\Lambda_{\tau, q}$ for the Lorenz-63 model and observable $\phi_{\text {con }}$ : box plots as a function of lead time $\tau$ for different thresholds $q$. Boxes indicate the interquartile range, whiskers extend to the minimum and maximum values, and circles mark the median. For each lead time, four thresholds are chosen: $q=-\infty$ (grey) and the 95th, 97th, and 99th percentiles of the observable (red, green, and blue, respectively).

\section{Methodology}

In summary, our methodology consists of checking whether initial conditions leading to extreme values typically have larger or smaller finite-time Lyapunov exponents.

\subsection{Extreme events}

The setting is a system of ordinary differential equations

$\dot{x}=f(x), \quad x \in \mathbb{R}^{n}$,

and we denote the evolution operator by $\Phi_{t}$. In addition, we consider a scalar observable $\phi: \mathbb{R}^{n} \rightarrow \mathbb{R}$. We define the extreme value domain as the set of points in phase space for which the observable exceeds a threshold $q$ :

$E_{q}=\left\{x \in \mathbb{R}^{n}: \phi(x)>q\right\}$.

We speak of an extreme event whenever a trajectory $\Phi_{t}(x)$ enters the set $E_{q}$.

The papers on extreme value theory for dynamical systems cited in the introduction focused on the case where $\phi$ has the form

$\phi(x)=g(\operatorname{dist}(x, \tilde{x}))$,

where $g:[0, \infty) \rightarrow \mathbb{R}$ is a continuous function and $\tilde{x}$ is a point on the attractor. However, as pointed out by Holland et al. (2012b), physically relevant observations are not of this form. For example, geophysical quantities such as vorticity or components of the velocity vector can often be written as

$\phi(x)=q^{\top} x$,

where $q \in \mathbb{R}^{n}$ is a nonzero vector. Positive quantities, such as energy, enstrophy, or squared wind speeds can often be written as

$\phi(x)=x^{\top} Q x$, where $Q=Q^{\top}$ is a nonnegative definite $n \times n$ matrix. In this paper we exclusively focus on observations of the form Eqs. (2) and (3).

\subsection{Finite-time Lyapunov exponents}

Infinitesimal errors in the initial condition $x$ of the system Eq. (1) in the direction $v$ evolve as $L_{t}(x) v$, where the matrix $L_{t}(x)$ is the solution of the matrix intial value problem

$\dot{X}=D f\left(\Phi_{t}(x)\right) X, \quad X(0)=I$,

which is often referred to as the tangent linear equation or first variational equation. The singular vectors of $L_{t}(x)$ give the direction and magnitude of maximal error growth along a finite-time segment of an orbit in state space (Buizza and Palmer, 1995; Palmer et al., 1998; Leutbecher and Palmer, 2008).

To measure the growth of perturbations over a time interval $[0, \tau]$, we use different norms at $t=0$ and $t=\tau$. Let $C$ be the initial error covariance matrix, and let $P$ be an orthogonal projection matrix (i.e. $P=P^{2}=P^{\top}$ ). Let $\|\cdot\|$ denote the Euclidean norm. The Rayleigh-Ritz quotient

$$
\frac{\left\|P L_{\tau}(x) v\right\|^{2}}{\left\|C^{-1 / 2} v\right\|^{2}}=\frac{v^{\top} L_{\tau}(x)^{\top} P L_{\tau}(x) v}{v^{\top} C^{-1} v}
$$

is maximal if and only if $v$ is the right singular vector of $P L_{\tau}(x) C^{1 / 2}$ associated with the largest singular value. We define the time- $\tau$ finite-time Lyapunov exponents (FTLE) as

$\lambda_{i}(x, \tau)=\frac{1}{\tau} \log \sigma_{i}(x, \tau), \quad i=1, \ldots, n$,

where $\sigma_{1}(x, \tau) \geq \sigma_{2}(x, \tau) \geq \cdots \geq \sigma_{n}(x, \tau)$ are the singular values of $P L_{\tau}(x) C^{1 / 2}$. In the following we assume that the initial error covariance $C$ is the identity matrix. 
Table 1. Descriptive statistics for the observables used with the three models.

\begin{tabular}{llrrrrrr}
\hline Model & Observable & Min & Mean & Max & 95th percentile & 97th percentile & 99th percentile \\
\hline Lorenz-63 & $\phi_{\text {con }}$ & -19.13 & 0.04 & 19.09 & 13.34 & 14.27 & 15.66 \\
& $\phi_{\text {en }}$ & 9.57 & 772.42 & 2630.22 & 1744.66 & 1859.84 & 2058.29 \\
\hline \multirow{2}{*}{ Barotropic vorticity } & $\phi_{\text {sid }}$ & 235.36 & 659.17 & 960.70 & 879.78 & 891.16 & 928.99 \\
equation & $\phi_{\text {top }}$ & 0.00 & 43.28 & 1044.14 & 191.44 & 254.23 & 406.08 \\
& $\phi_{\text {val }}$ & 2.13 & 430.42 & 974.70 & 711.20 & 757.27 & 851.57 \\
\hline Lorenz-96 & $\phi_{\text {en }}$ & 399.40 & 674.20 & 1009.50 & 795.50 & 814.20 & 849.50 \\
\hline
\end{tabular}

We choose the projection matrix $P$ such that the singular vectors will point in the direction of maximal growth of the observable $\phi$. For an observable of the form Eq. (2), we set

$P=\frac{q q^{\top}}{\|q\|^{2}}$

so that

$\|P x\|^{2}=\frac{\phi(x)^{2}}{\|q\|^{2}}$.

In case of observable Eq. (3), recall that a symmetric nonnegative definite $n \times n$ matrix has $k \leq n$ positive eigenvalues $\mu_{1} \geq \cdots \geq \mu_{k}>0$ and the corresponding eigenvectors $q_{i}$ are orthonormal. We can rewrite Eq. (3) as

$\phi(x)=x^{\top} Q x=\sum_{i=1}^{k} \mu_{i}\left(q_{i}^{\top} x\right)^{2}$.

In this case we set

$P=\sum_{i=1}^{k} q_{i} q_{i}^{\top}$

so that

$\|P x\|^{2}=\sum_{i=1}^{k}\left(q_{i}^{\top} x\right)^{2}$,

and

$\mu_{k}\|P x\|^{2} \leq \phi(x) \leq \mu_{1}\|P x\|^{2}$.

Hence, the $\phi(x)$ is large if and only if $\|P x\|$ is large.

\subsection{Predictability of extreme values}

We fix an initial point $x_{0}$ on the attractor and a sampling frequency $\omega>0$ and compute a sample of points along an orbit on the attractor:

$S=\left\{\Phi_{k / \omega}\left(x_{0}\right) \mid k=1, \ldots, N\right\}$.

We choose $\omega$ and $N$ sufficiently large to provide a good sampling of the attractor as far as both local and global fluctuations are concerned. Typically, we take $N=10^{6}$, whereas $\omega$ depends on the time scale of the system of interest.
To study the predictability of extreme events, we define the set

$\Lambda_{\tau, q}=\left\{\lambda_{1}(x, \tau) \mid x \in S\right.$ and $\left.\Phi_{\tau}(x) \in E_{q}\right\}$

containing the FTLEs for initial conditions such that after $\tau$ units of time the observable exceeds the threshold $q$. We study how the distribution of this set changes as the lead time $\tau$ and threshold $q$ increase. For fixed lead times we compare the distributions of the set $\Lambda_{\tau, q}$ for $q=-\infty$ (the entire sample) and for $q$ being the 95th, 97th, and 99th percentiles of the sample

$\{\phi(x) \mid x \in S\}$.

If FTLEs cluster to larger positive (smaller negative) values when $q$ increases, then extremes are less (better) predictable.

\section{Results}

We apply the methodology of Sect. 2 to three geophysical models: the Lorenz-63 and Lorenz-96 models and a spectral truncation of the barotropic vorticity equation. As observations we will take energy, convection, and squared wind speeds.

\subsection{The Lorenz-63 model}

In this section we consider the classical model for RayleighBénard convection derived by Lorenz (1963):

$\dot{x}=\sigma(y-x), \quad \dot{y}=x(\rho-z)-y, \quad \dot{z}=x y-\beta z$,

with the classical parameter values $\sigma=10, \rho=28$, and $\beta=$ $8 / 3$. We use the observables

$\phi_{\mathrm{con}}(x, y, z)=x \quad$ and $\quad \phi_{\mathrm{en}}(x, y, z)=x^{2}+y^{2}+z^{2}$

that respectively measure the intensity of convection and the total energy. For the projection matrix in Eq. (5), we respectively take

$P_{\text {con }}=\left(\begin{array}{lll}1 & 0 & 0 \\ 0 & 0 & 0 \\ 0 & 0 & 0\end{array}\right)$ and $\quad P_{\mathrm{en}}=\left(\begin{array}{lll}1 & 0 & 0 \\ 0 & 1 & 0 \\ 0 & 0 & 1\end{array}\right)$. 


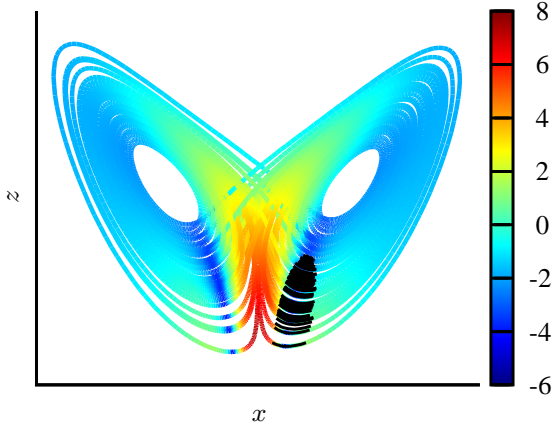

(a) $\tau=0.25$

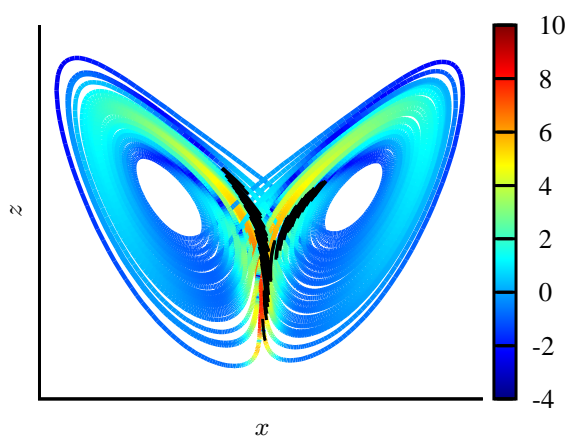

(b) $\tau=0.5$

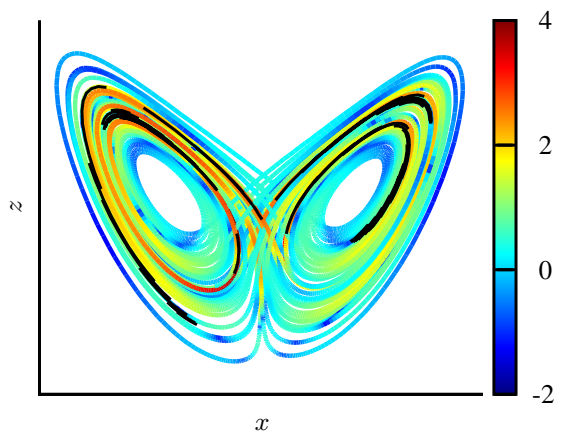

(c) $\tau=1.5$

Fig. 2. Projection of the Lorenz-63 attractor on the $(x, z)$-plane with colours indicating the magnitude of the maximal time- $\tau$ FTLE. Black dots mark initial conditions leading to an exceedance of the 95th percentile of the observable $\phi_{\text {con }}$. For $\tau=0.25$, initial conditions leading to extremes typically have low FTLE, but for $\tau=0.25$, they typically have a large FTLE. This difference vanishes for longer lead times.

By numerical integration we computed a sample of $N=10^{6}$ points on the attractor using the sample frequency $\omega=100$. Table 1 lists some statistics of the observables evaluated at the sample points.

Figure 1 shows how the distribution of the set $\Lambda_{\tau, q}$ changes with lead time and threshold $q$ for the observable $\phi_{\text {con. }}$. For $\tau \leq 0.25$ more than 75 percent of the inital conditions leading to an exceedance of the 99th percentile have a

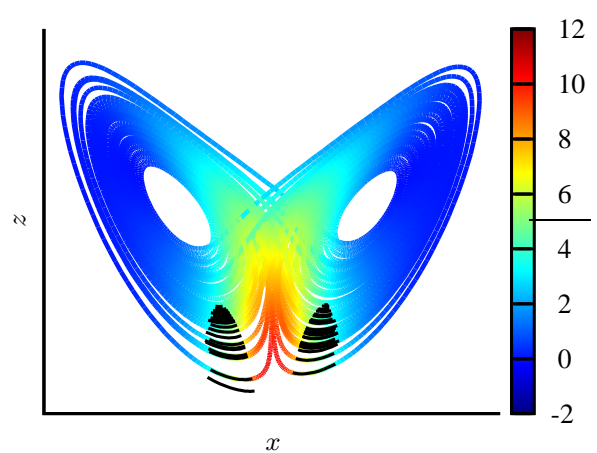

(a) $\tau=0.25$

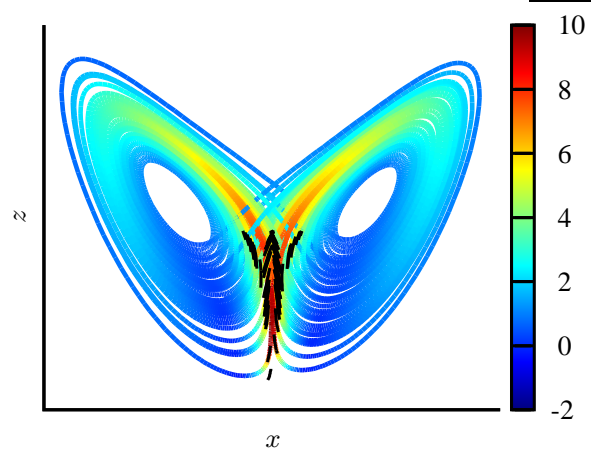

(b) $\tau=0.5$

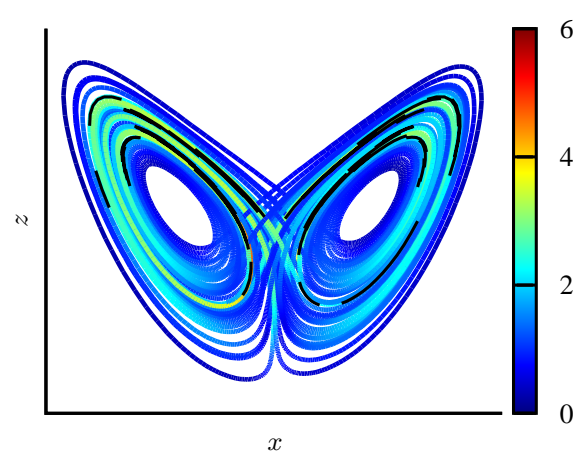

(c) $\tau=1.5$

Fig. 3. As Fig. 2, but for the observable $\phi_{\text {en }}$. Initial conditions leading to extremes typically also have a large FTLE, and this persists for larger lead times.

negative FTLE. This suggest that extreme values of convection are well-predictable up to small lead times. For $\tau>0.25$ more than 75 percent of the initial conditions leading to an exceedance of the 99th quantile have a FTLE that is larger than the median of $\Lambda_{\tau,-\infty}$, which indicates that extreme values for this lead time are less predictable. When the lead time $\tau$ increases, the distributions of $\Lambda_{\tau, q}$ for different thresholds $q$ become similar; this suggests that the difference in predictability between extremes and non-extremes disappears. 


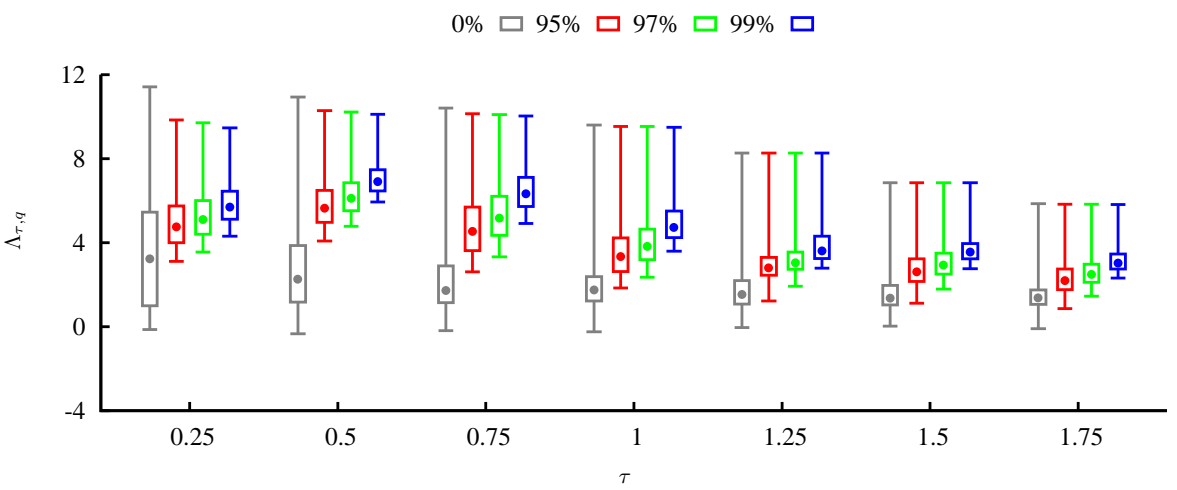

Fig. 4. As Fig. 1, but for the observable $\phi_{\mathrm{en}}$.

Figure 4 shows how the distribution of the set $\Lambda_{\tau, q}$ changes with increasing lead time and threshold for the observable $\phi_{\text {en }}$. For all lead times $\tau$ almost all initial conditions leading to an exceedance of the 95th percentile of the energy have a FTLE that is larger than the median of $\Lambda_{\tau,-\infty}$. This means that in this case extreme values of the energy observable $\phi_{\mathrm{en}}$ are systematically less predictable than non-extreme values. Only for lead times much longer than $\tau=1.75$, the distributions of $\Lambda_{\tau, q}$ for different quantiles $q$ become similar (not shown).

Figures 2 and 3 show how for the observables $\phi_{\text {con }}$ and $\phi_{\mathrm{en}}$ the FTLEs vary on different parts on the attractor for various lead times. Black dots mark the initial conditions which lead to an exceedance of the 95th percentile within the stated lead time. For short lead times, initial conditions with large FTLE are located in the central region of the attractor where the two wings of the butterfly-shaped attractor cross each other. When the lead time increases the unpredictable regions quickly spread out on the entire attractor. The initial conditions leading to extreme energy values systematically coincide with the parts on the attractor having larger FTLEs, whereas initial conditions leading to extreme convection do not.

\subsection{The barotropic vorticity equation}

In this section we consider the barotropic vorticity equation (BVE) for atmospheric flow over orography:

$$
\frac{\partial}{\partial t} \Delta \psi=-J(\psi, \Delta \psi+\beta y+\gamma h)-C \Delta\left(\psi-\psi^{*}\right),
$$

where $\psi$ is the stream function, $\psi^{*}$ is the forcing, $\beta y$ is the Coriolis force, $h$ is the orography, and $\Delta$ is the Laplace operator. In addition, $J$ is the Jacobian operator:
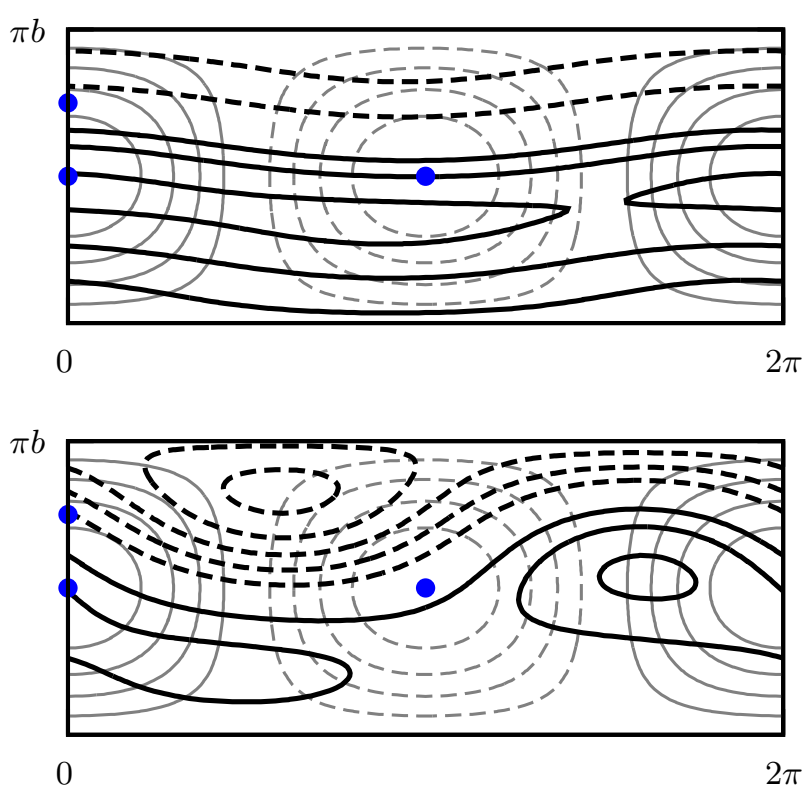

Fig. 5. Contour plots of the stream function fields of the zonal regime (top) and blocked regime (bottom) in the barotropic vorticity Eq. (8). Blue dots mark the locations of the three wind speed observables (see main text).

$J(f, g)=\frac{\partial f}{\partial x} \frac{\partial g}{\partial y}-\frac{\partial f}{\partial y} \frac{\partial g}{\partial x}$.

The domain is $[0,2 \pi] \times[0, \pi b]$, where $b$ equals twice the aspect ratio of the $\beta$-plane channel. We take periodic boundary conditions in $x$ and for $y=0, \pi b$, we require

$\frac{\partial \psi}{\partial x}=0, \quad \int_{0}^{2 \pi} \frac{\partial \psi}{\partial y} d x=0$, 
Table 2. Parameters and coefficients of the model Eq. (8) as used by Crommelin et al. (2004).

\begin{tabular}{llllll}
\hline Par & Value & Par & Value & Par & Value \\
\hline$C$ & 0.1 & $\alpha_{2}$ & $32 \sqrt{2}\left(b^{2}+3\right) / 15 \pi\left(b^{2}+4\right)$ & $\gamma$ & 0.2 \\
$b$ & 0.5 & $\beta$ & $5 / 4$ & $\gamma_{1}$ & $4 \sqrt{2} b \gamma / 3 \pi\left(b^{2}+1\right)$ \\
$\hat{\psi}_{1}^{*}$ & 0.95 & $\beta_{1}$ & $\beta b^{2} /\left(b^{2}+1\right)$ & $\gamma_{2}$ & $32 \sqrt{2} b \gamma / 15 \pi\left(b^{2}+4\right)$ \\
$r$ & -0.801 & $\beta_{2}$ & $\beta b^{2} /\left(b^{2}+4\right)$ & $\tilde{\gamma}_{1}$ & $4 \sqrt{2} b \gamma / 3 \pi$ \\
$\hat{\psi}_{4}^{*}$ & $r \hat{\psi}_{1}^{*}$ & $\delta_{1}$ & $64 \sqrt{2} b^{2} / 15 \pi\left(b^{2}+1\right)$ & $\tilde{\gamma}_{2}$ & $8 \sqrt{2} b \gamma / 15 \pi$ \\
$\alpha_{1}$ & $8 \sqrt{2} b^{2} / 3 \pi\left(b^{2}+1\right)$ & $\delta_{2}$ & $64 \sqrt{2}\left(b^{2}-3\right) / 15 \pi\left(b^{2}+4\right)$ & $\varepsilon$ & $16 \sqrt{2} / 5 \pi$ \\
\hline
\end{tabular}

$0 \% \square 95 \% \square 97 \% \square 99 \% \square$

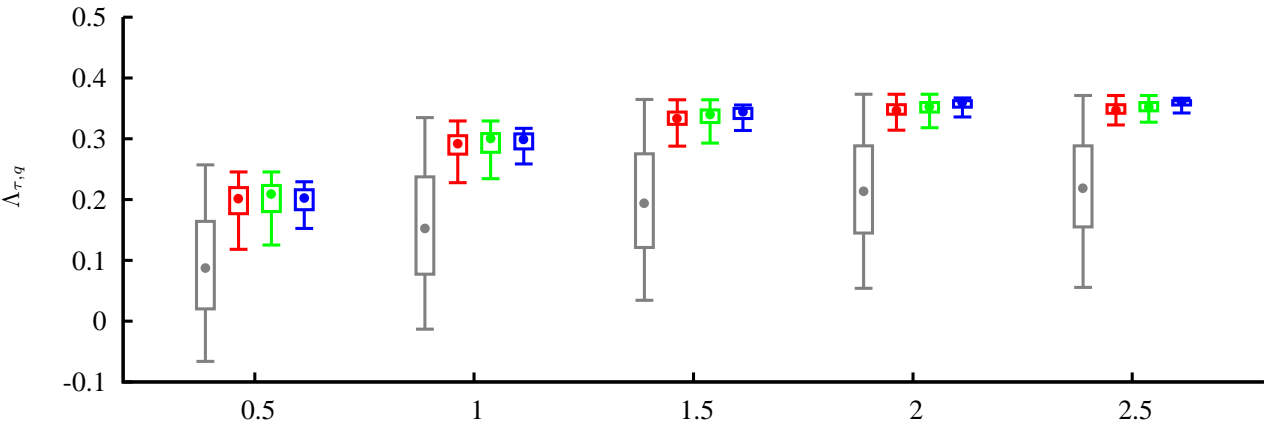

Fig. 6. As Fig. 1, but for the model Eq. (8) with observable $\phi_{\text {sid }}$.

which respectively mean that on the lateral boundaries the northward component of the velocity vanishes and that the net zonal flow is zero.

Charney and DeVore (1979) studied a 3-dimensional spectral truncation of Eq. (7), and detected coexisting equilibria representing blocked and zonal flow patterns. De Swart (1989) studied higher order truncations, including the 6dimensional truncation

$$
\begin{aligned}
& \dot{\hat{\psi}}_{1}=\tilde{\gamma}_{1} \hat{\psi}_{3}-C\left(\hat{\psi}_{1}-\hat{\psi}_{1}^{*}\right), \\
& \dot{\hat{\psi}}_{2}=-\left(\alpha_{1} \hat{\psi}_{1}-\beta_{1}\right) \hat{\psi}_{3}-C \hat{\psi}_{2}-\delta_{1} \hat{\psi}_{4} \hat{\psi}_{6}, \\
& \dot{\hat{\psi}}_{3}=\left(\alpha_{1} \hat{\psi}_{1}-\beta_{1}\right) \hat{\psi}_{2}-\gamma_{1} \hat{\psi}_{1}-C \hat{\psi}_{3}+\delta_{1} \hat{\psi}_{4} \hat{\psi}_{5}, \\
& \dot{\hat{\psi}}_{4}=\tilde{\gamma}_{2} \hat{\psi}_{6}-C\left(\hat{\psi}_{4}-\hat{\psi}_{4}^{*}\right)+\varepsilon\left(\hat{\psi}_{2} \hat{\psi}_{6}-\hat{\psi}_{3} \hat{\psi}_{5}\right), \\
& \dot{\hat{\psi}}_{5}=-\left(\alpha_{2} \hat{\psi}_{1}-\beta_{2}\right) \hat{\psi}_{6}-C \hat{\psi}_{5}-\delta_{2} \hat{\psi}_{4} \hat{\psi}_{3}, \\
& \dot{\hat{\psi}}_{6}=\left(\alpha_{2} \hat{\psi}_{1}-\beta_{2}\right) \hat{\psi}_{5}-\gamma_{2} \hat{\psi}_{4}-C \hat{\psi}_{6}+\delta_{2} \hat{\psi}_{4} \hat{\psi}_{2} .
\end{aligned}
$$

We study this model with the parameters of Crommelin et al. (2004), which are listed in Table 2. With this choice the channel $[0,2 \pi] \times[0, \pi b]$ has dimensions $5000 \times 1250 \mathrm{~km}$, the orography $h(x, y)=\cos (x) \sin (y / b)$ has a dimensional amplitude of $200 \mathrm{~m}$, and one unit of time corresponds to one day.

Crommelin et al. (2004) found intermittent transitions between two regions in state space corresponding to zonal and blocked flows, see Fig. 5. The dynamics consists of three recurrent episodes: (1) transitions from zonal to blocked flows, typically taking 30 days, (2) transitions from blocked to zonal flows, typically taking 40-80 days, and (3) spiraling behaviour around the zonal regime, typically lasting more than 200 days. This intermittent behaviour can be explained by the presence of Shil'nikov-like strange attractors, due to homoclinic bifurcations taking place near a Hopf-saddle-node bifurcation (Broer and Vegter, 1984; Broer and Vitolo, 2008).

As observable we use the squared wind speed at several locations in the $\beta$-plane channel. In Appendix A we show that the east- and northward components of the wind vector at $(x, y)$ can be computed from the state vector $\hat{\psi}$ as $q_{1}^{\top} \hat{\psi}$ and $q_{2}^{\top} \hat{\psi}$, where $q_{1}, q_{2} \in \mathbb{R}^{6}$ are orthogonal vectors depending on $(x, y)$. Hence, the squared wind speed at location $(x, y)$ is given by

$\phi(\hat{\psi})=\left(q_{1}^{\top} \hat{\psi}\right)^{2}+\left(q_{2}^{\top} \hat{\psi}\right)^{2}=\hat{\psi}^{\top}\left(q_{1} q_{1}^{\top}+q_{2} q_{2}^{\top}\right) \hat{\psi}$.

For the projection matrix in Eq. (5) we take

$P=\frac{q_{1} q_{1}^{\top}}{\left\|q_{1}\right\|^{2}}+\frac{q_{2} q_{2}^{\top}}{\left\|q_{2}\right\|^{2}}$.

We will study the extreme wind speeds at three locations: the side of the orography $(x, y)=(0,3 \pi b / 4)$, the top $(x, y)=$ $(0, \pi b / 2)$, and the valley $(x, y)=(\pi, \pi b / 2)$, see Fig. 5 . The corresponding wind speed observables are denoted by $\phi_{\text {side }}$, $\phi_{\text {top }}$, and $\phi_{\mathrm{val}}$. By numerical integration we computed a sample of $N=10^{6}$ points on the attractor using the sample frequency $\omega=2$. Table 1 lists some statistics of the observables evaluated at the sample points. 


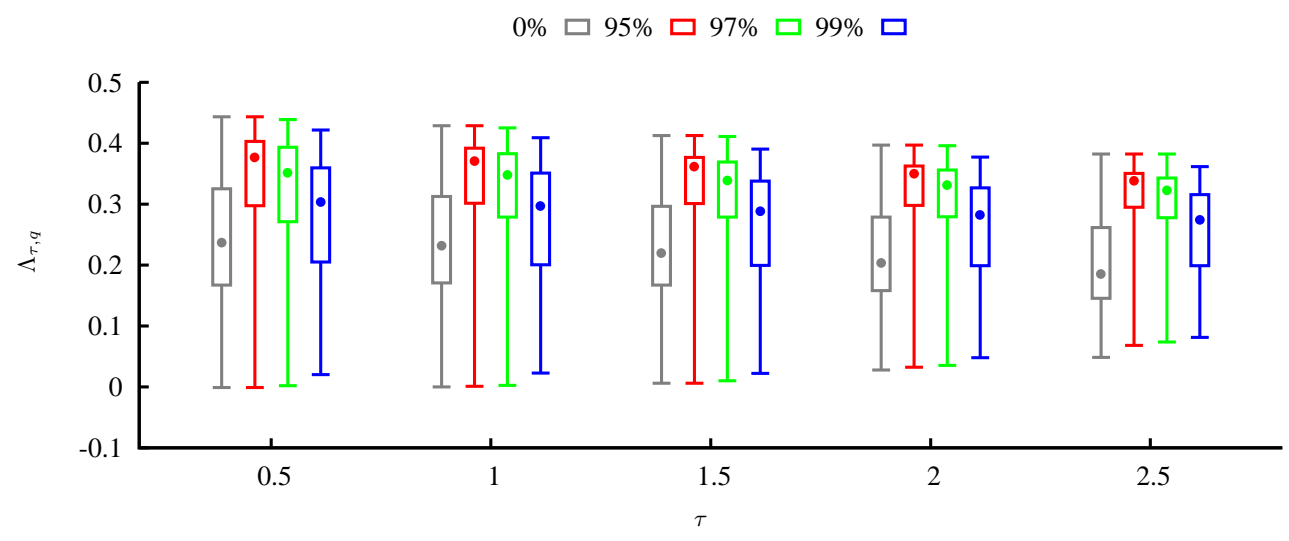

Fig. 7. As Fig. 6, but for the observable $\phi_{\text {top. }}$.

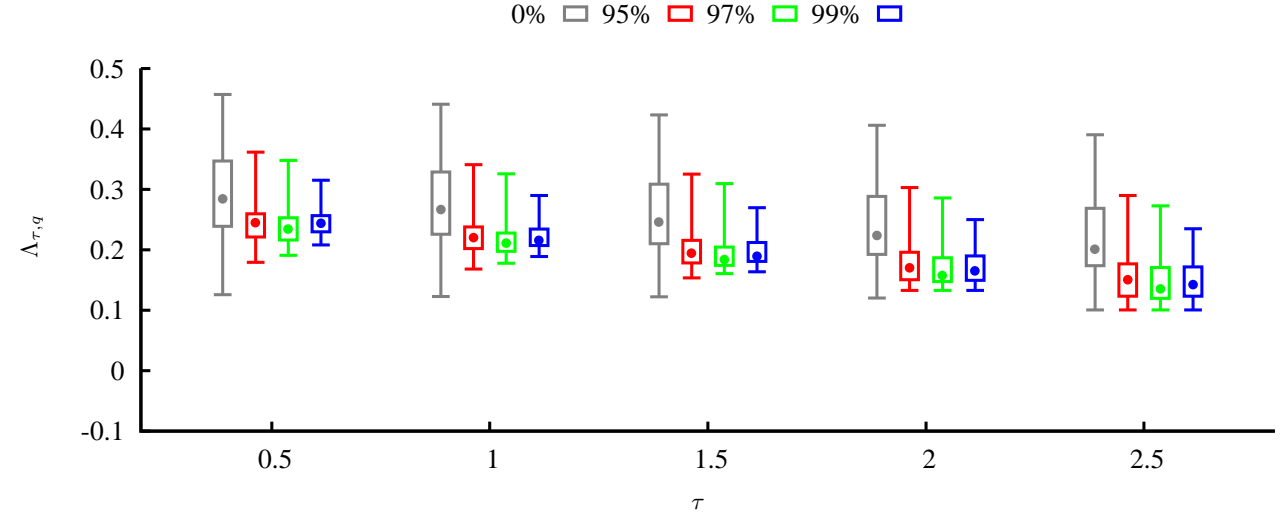

Fig. 8. As Fig. 6, but for the observable $\phi_{\text {val }}$.

Figures 6,7 , and 8 show how the distributions of the set $\Lambda_{\tau, q}$ change with lead time $\tau$ and threshold $q$ for three observables $\phi_{\text {sid }}, \phi_{\text {top }}$, and $\phi_{\text {val }}$. For $\phi_{\text {sid }}$ the initial conditions leading to extremes systematically have a large FTLE, which means that extremes are less predictable. For $\phi_{\text {top }}$ the FTLEs for initial conditions leading to extremes are highly variable. In this case one cannot say that extremes are either better or less predictable than generic events. Finally, for $\phi_{\text {val }}$ the FTLEs for initial conditions leading to extremes are systematically low: more than $75 \%$ of the initial conditions leading to extremes have an FTLE smaller than the median FTLE for all events.

Figures 9-11 show the FTLEs vary on different parts of the attractor. For all observables the initial conditions with large FTLE are located near the zonal regime and the initial conditions with small FTLE are located near the blocked regime. This separation persists remarkably for increasing lead time. This is very different from the Lorenz-63 attractor, for which the unpredictable regions quickly spread out along the attractor. For the observables $\phi_{\text {sid }}$ and $\phi_{\text {top }}$ the initial conditions leading to extreme wind speeds systematically coincide with the unpredictable parts of the attractor. For $\phi_{\mathrm{val}}$ the initial conditions leading to extremes intersect the more predictable regions on the attractor.

\subsection{The Lorenz-96 model}

The Lorenz-96 model is a conceptual model for traveling waves in the atmosphere (Lorenz, 1996). This model is used to test data assimilation methods (Trevisan and Palatella, 2011) and subgrid scale parameterizations (Crommelin and Vanden-Eijnden, 2008). Although the model is not derived from physical principles it has features which are typical for geophysical models: forcing, dissipation, and energy preserving quadratic terms.

The model variables $x_{1}, \ldots, x_{n}$ can be interpreted as meteorological quantities, such as pressure or vorticity, along a circle of constant latitude where the index $i$ of each variable $x_{i}$ plays the role of longitude. The dynamical equations are

$$
\frac{d x_{i}}{d t}=x_{i-1}\left(x_{i+1}-x_{i-2}\right)-x_{i}+F, \quad i=1, \ldots, n>3,
$$




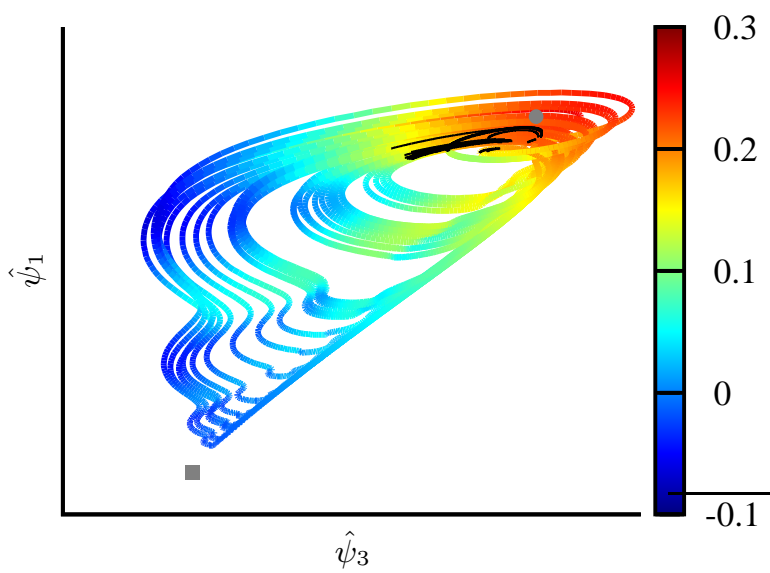

(a) $\tau=0.5$

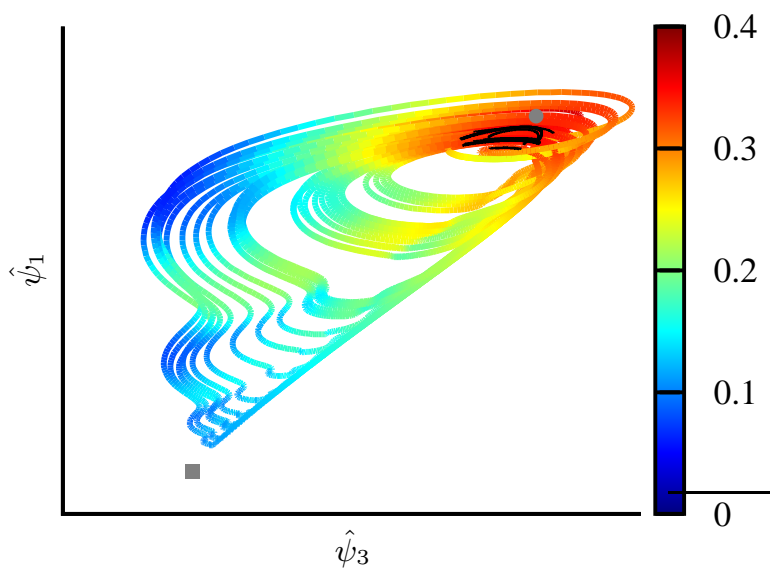

(b) $\tau=2.5$

Fig. 9. Projection on the $\left(\widehat{\psi}_{3}, \widehat{\psi}_{1}\right)$-plane of a the attractor of Eq. (8) with colour indicating the magnitude of the maximal time- $\tau$ FTLE. The grey circle (square) indicates the point in phase space corresponding with the zonal (blocked) regime of Fig. 5. Black dots mark initial conditions leading to an exceedance of the 95 th percentile of the observable $\phi_{\text {sid }}$. Note that initial conditions leading to high wind speeds are located near the zonal regime, where also the FTLEs are large.

with periodic "boundary conditions" $x_{i+n}=x_{i}$. The dimension $n$ and forcing $F>0$ are free parameters. We set $n=36$ and $F=8$, which were originally used by Lorenz.

We use the observable

$\phi_{\mathrm{en}}(x)=\sum_{i=1}^{n} x_{i}^{2}$

which measures the total energy of the system. For the projection in Eq. (5), we take the identity matrix. By numerical integration we computed a sample of $N=10^{6}$ points on the attractor using the sample frequency $\omega=100$. Table 1

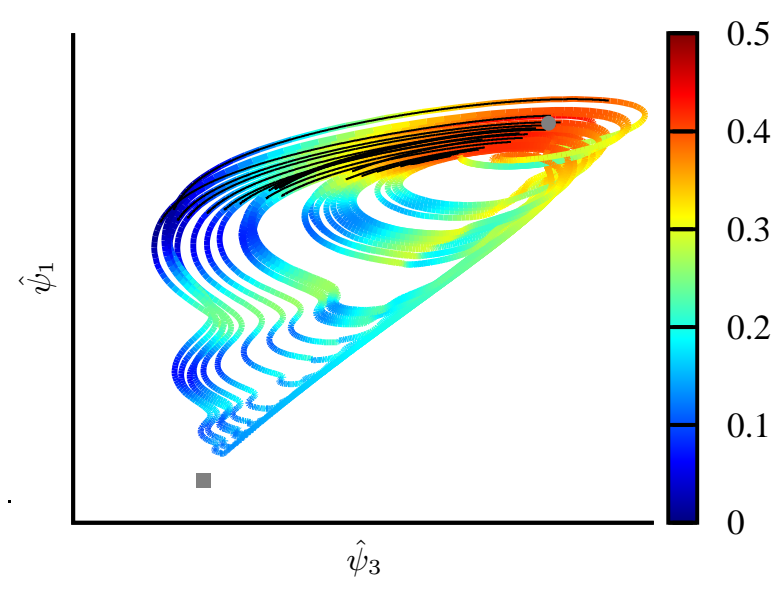

(a) $\tau=0.5$

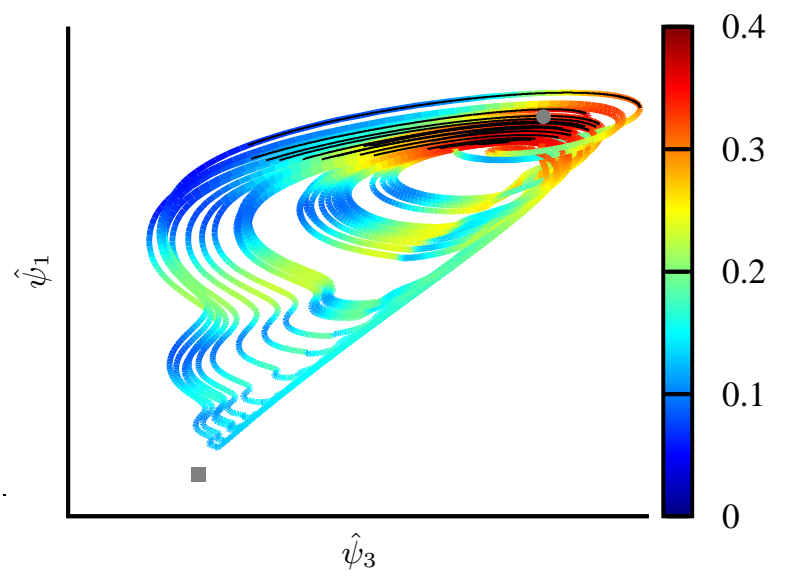

(b) $\tau=2.5$

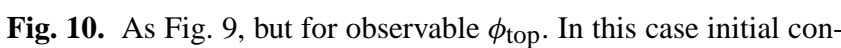
ditions leading to large wind speeds are not confined to the unpredictable region of the attractor.

lists some statistics of the observable evaluated at the sample points.

Figure 12 shows how the distribution of the set $\Lambda_{\tau, q}$ changes with lead time $\tau$ and threshold $q$. For all lead times $\tau$, the distribution of the maximal FTLE does not change very much with increasing threshold $q$. This suggests that extreme values of the energy observable are neither better nor less predictable than non-extreme values.

\section{Conclusions and discussion}

In this paper we investigated the predictability of extreme values in geophysical models. We studied how FTLEs depend on forecast lead time and the threshold on the observable. General statements on whether extreme values are better or worse to predict are not possible. Whether initial 


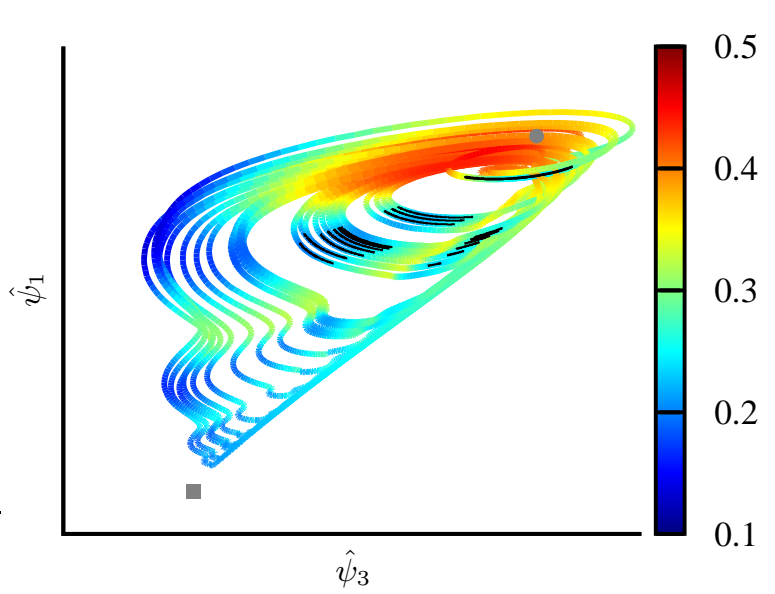

(a) $\tau=0.5$

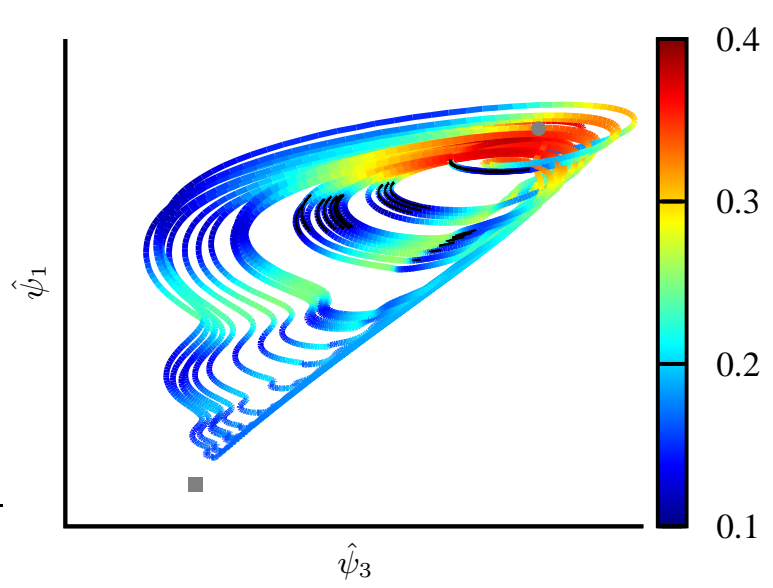

(b) $\tau=2.5$

Fig. 11. As Fig. 9, but for observable $\phi_{\text {val }}$. In this case initial conditions leading to large wind speeds are located in the more predictable regions of the attractor.

conditions leading to extreme values have larger FTLEs depends on (1) the observable, (2) the attractor of the system, and (3) the prediction lead time. Table 3 presents the main conclusion for each model/observable pair.

FTLEs measure forecast error growth rate under the assumption that errors in the initial condition are infinitesimally small. For finite-size, but small, errors, the maximal FTLE still is a good estimate of error growth (Harle et al., 2006). For larger errors, however, error growth may no longer be exponential and this will also effect the predictability of extreme values. The present study should, therefore, be extended to the setting of ensemble forecasts in which the predictability of extremes is measured in terms of the dispersion of ensemble members. This approach would also be more appropriate for operational weather forecasting models without a tangent linear model needed to compute FTLEs, such as
Table 3. The predictability of extreme values for the three models with different observables.

\begin{tabular}{lll}
\hline Model & Obs. & Predictability of extremes \\
\hline Lorenz-63 & $\phi_{\text {con }}$ & $\begin{array}{l}\text { For lead times up to } \tau=0.25 \text { extremes } \\
\text { are well-predictable (negative FTLEs), } \\
\text { but for longer lead times they are just as } \\
\text { predictable as non-extremes. }\end{array}$ \\
& $\phi_{\text {en }}$ & $\begin{array}{l}\text { For lead times up to } \tau=01.75 \text { extremes } \\
\text { are less predictable than non-extremes. }\end{array}$ \\
\hline BVE & $\phi_{\text {sid }}$ & $\begin{array}{l}\text { Extremes are less predictable, and this } \\
\text { persists up to long lead times due to the } \\
\text { intermittent nature of the dynamics. } \\
\end{array}$ \\
& $\phi_{\text {top }}$ & $\begin{array}{l}\text { Extremes are neither better nor less } \\
\text { predictable than non-extremes. } \\
\text { Extremes are better predictable than } \\
\text { non-extremes }\end{array}$ \\
\hline Lorenz-96 & $\phi_{\text {en }}$ & $\begin{array}{l}\text { Extremes are neither better nor less } \\
\text { predictable than non-extremes. }\end{array}$ \\
\hline
\end{tabular}

the MOGREPS system of the UK Met Office (Bowler et al., 2008).

Another important question is: how predictable are realworld extremes, such as wind storms? Large-scale flow patterns, such as the North Atlantic Oscillation, cause temporal clustering of storms (Mailier et al., 2006; Vitolo et al., 2009). The emergence of these patterns might be a manifestation of intermittency, i.e. the irregular alternation between phases of chaotic and non-chaotic, such as steady or periodic dynamics (Pomeau and Manneville, 1980). For example, the spectral truncation Eq. (8) of the barotropic vorticity equation exhibits intermittent transitions between zonal and blocked flows due to Shil'nikov-like strange attractors appearing near a Hopf-saddle-node bifurcation (Crommelin et al., 2004; Broer and Vitolo, 2008; Broer and Vegter, 1984). Other forms of intermittency due to bifurcations of planetary waves have been detected in low-order models of the shallow water equations (Sterk et al., 2010). Because different intermittent phases can have different error growth rates, the emergence of large-scale flow patterns might enhance predictability of extremes.

Finally, we note that FTLEs might not be the best measures of finite-time predictability. A different approach would be to apply techniques based on Takens' reconstruction theorem, such as correlation integrals and entropy; see Broer and Takens (2011) and references therein. Such techniques have been applied to develop early warning systems for thermal excursions in chemical reactors (Zaldívar et al., 2005). Potentially, these techniques can be useful in the prediction of extreme values in geophysical applications. We believe that these questions and problems will have sufficient potential for future research. 
$0 \% \square 95 \% \square 97 \% \square 99 \% \square$

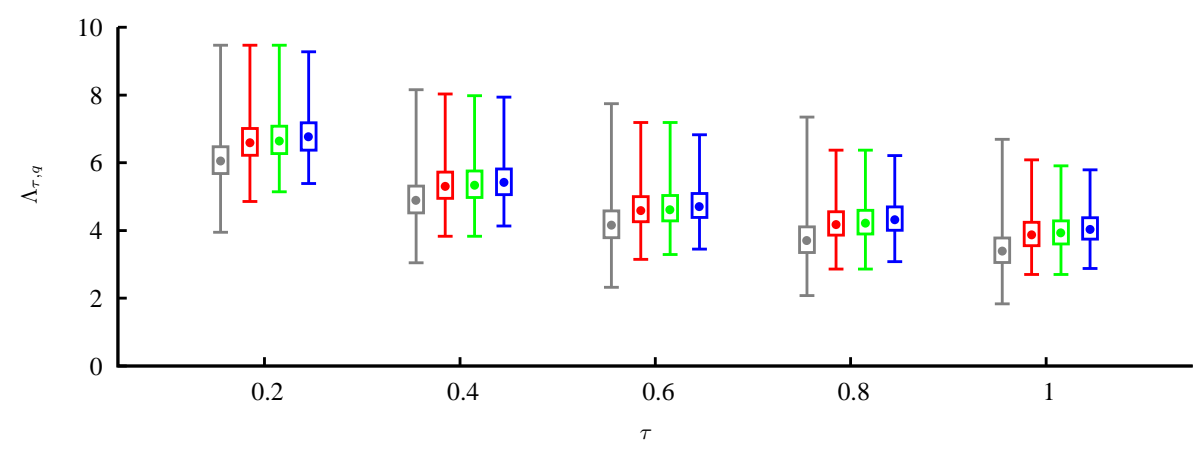

Fig. 12. As Fig. 1, but for the Lorenz-96 model Eq. (9) with observable $\phi_{\mathrm{en}}$.

\section{Appendix A}

\section{Wind speed in the barotropic vorticity equation}

The stream function can be computed from the spectral coefficients $\hat{\psi}_{i}$ by

$$
\begin{aligned}
\psi(x, y)=b \sqrt{2} \hat{\psi}_{1} \cos (y / b)+b \sqrt{2} \hat{\psi}_{4} \cos (2 y / b) \\
+2 b\left\{\hat{\psi}_{2} \cos (x)+\hat{\psi}_{3} \sin (x)\right\} \sin (y / b) \\
+2 b\left\{\hat{\psi}_{5} \cos (x)+\hat{\psi}_{6} \sin (x)\right\} \sin (2 y / b) .
\end{aligned}
$$

De Swart (1988) uses the convention $u=-\psi_{y}, v=\psi_{x}$. This gives the following expressions for the east- and northward components of the velocity field:

$$
\begin{aligned}
u(x, y)=\sqrt{2} & \hat{\psi}_{1} \sin (y / b)+2 \sqrt{2} \hat{\psi}_{4} \sin (2 y / b) \\
- & 2\left\{\hat{\psi}_{2} \cos (x)+\hat{\psi}_{3} \sin (x)\right\} \cos (y / b) \\
- & 4\left\{\hat{\psi}_{5} \cos (x)+\hat{\psi}_{6} \sin (x)\right\} \cos (2 y / b), \\
v(x, y)=-2 b & \left\{\hat{\psi}_{2} \sin (x)-\hat{\psi}_{3} \cos (x)\right\} \sin (y / b) \\
& -2 b\left\{\hat{\psi}_{5} \sin (x)-\hat{\psi}_{6} \cos (x)\right\} \sin (2 y / b) .
\end{aligned}
$$

This can be rewritten as

$u(x, y)=q_{1}^{\top} \hat{\psi} \quad$ and $\quad v(x, y)=q_{2}^{\top} \hat{\psi}$,

where

$$
q_{1}=\left(\begin{array}{c}
\sqrt{2} \sin (y / b) \\
2 \cos (x) \cos (y / b) \\
2 \sin (x) \cos (y / b) \\
2 \sqrt{2} \sin (2 y / b) \\
-4 \cos (x) \cos (2 y / b) \\
-4 \sin (x) \cos (2 y / b)
\end{array}\right)
$$

and

$$
q_{2}=\left(\begin{array}{c}
0 \\
-2 b \sin (x) \sin (y / b) \\
2 b \cos (x) \sin (y / b) \\
0 \\
-2 b \sin (x) \sin (2 y / b) \\
2 b \cos (x) \sin (2 y / b)
\end{array}\right) .
$$

Note that the vectors $q_{1}$ and $q_{2}$ are orthogonal.

Acknowledgements. This research is part of the project "PREDEX: Predictability of Extreme Weather Events", funded by Complexity-NET: (www.complexitynet.eu). The authors gratefully acknowledge support by the UK and Dutch funding agencies involved in Complexity-NET: the EPSRC and the NWO. The authors are also indebted to their respective institutes for kind hospitality. Finally, the authors wish to thank Jan Barkmeijer, Theodoros Economou, Chris Ferro, Ken Mylne, and David Stephenson for useful discussions and remarks.

Edited by: S. Vannitsem

Reviewed by: two anonymous referees

\section{References}

Abarbanel, H., Brown, R., and Kennel, M.: Variation of Lyapunov Exponents on a Strange Attractor, J. Nonlin. Sci., 1, 175-199, 1991.

Bailey, B., Ellner, S., and Nychka, D.: Chaos with confidence: asymptotics and applications of local Lyapunov exponents, Fields Institute Communications, 11, 115-133, 1997.

Beirlant, J., Goegebeur, Y., Teugels, J., and Segers, J.: Statistics of Extremes: Theory and Applications, John Wiley and Sons, Berlin, 2004.

Bowler, N., Arribas, A., Mylne, K., Robertson, K., and Beare, S.: The MOGREPS short-range ensemble prediction system, Q. J. Roy. Meteorol. Soc., 134, 703-722, 2008.

Broer, H. and Takens, F.: Dynamical Systems and Chaos, 172, Appl. Math. Sci., Springer, 2011. 
Broer, H. and Vegter, G.: Subordinate Šil'nikov bifurcations near some singularities of vector fields having low codimension, Ergodic Theor. Dynam. Syst., 4, 509-525, 1984.

Broer, H. and Vitolo, R.: Dynamical systems modelling of lowfrequency variability in low-order atmospheric models, Discrete and Continuous Dynamical Systems B, 10, 401-419, 2008.

Buizza, R. and Palmer, T.: The singular-vector structure of the atmospheric global circulation, J. Atmos. Sci., 52, 1434-1456, 1995.

Charney, J. and DeVore, J.: Multiple Flow Equilibria in the Atmosphere and Blocking, J. Atmos. Sci., 36, 1205-1216, 1979.

Coles, S.: An Introduction to Statistical Modeling of Extreme Values, Springer Series in Statistics, Springer, New York, 2001.

Crommelin, D. and Vanden-Eijnden, E.: Subgrid scale parameterization with conditional Markov chains, J. Atmos. Sci., 65, 26612675, 2008.

Crommelin, D., Opsteegh, J., and Verhulst, F.: A Mechanism for Atmospheric Regime Behavior, J. Atmos. Sci., 61, 1406-1419, 2004.

Datta, S. and Ramaswamy, R.: Non-Gaussian fluctuations of local Lyapunov exponents at intermittency, J. Stat. Phys., 113, 283295, 2003.

De Swart, H.: Low-Order Spectral Models of the Atmospheric Circulation: A Survey, Acta Applicandae Mathematicae, 11, 49-96, 1988.

De Swart, H.: Analysis of a six-component atmospheric spectral model: chaos, predictability and vacillation, Physica D, 36, 222234, 1989.

Felici, M., Lucarini, V., Speranza, A., and Vitolo, R.: Extreme Value Statistics of the Total Energy in an Intermediate-Complexity Model of the Midlatitude Atmospheric Jet. Part I: Stationary Case, J. Atmos. Sci., 64, 2137-2158, 2007a.

Felici, M., Lucarini, V., Speranza, A., and Vitolo, R.: Extreme Value Statistics of the Total Energy in an Intermediate-Complexity Model of the Midlatitude Atmospheric Jet. Part II: Trend Detection and Assessment, J. Atmos. Sci., 64, 2159-2175, $2007 \mathrm{~b}$.

Freitas, A. and Freitas, J.: On the link between dependence and independence in extreme value theory for dynamical systems, Stat. Probab. Lett., 78, 1088-1093, 2008.

Freitas, A., Freitas, J., and Todd, M.: Extreme Value Laws in Dynamical Systems for Non-smooth Observations, J. Stat. Phys., 142, 108-126, 2010.

Gupta, C.: Extreme-value distributions for some classes of nonuniformly partially hyperbolic dynamical systems, Ergodic Theor. Dynam. Syst., 30, 757-771, 2010.

Haiman, G.: Extreme values of the tent map process, Stat. Probab. Lett., 65, 451-456, 2003.

Harle, M., Kwasniok, F., and Feudel, U.: Growth of finite errors in ensemble prediction, Nonlin. Processes Geophys., 13, 167-176, doi:10.5194/npg-13-167-2006, 2006.

Holland, M., Nicol, M., and Török, A.: Extreme value theory for non-uniformly expanding dynamical systems, T. Am. Math. Soc., 364, 661-688, 2012a.
Holland, M., Vitolo, R., Rabassa, P., Sterk, A., and Broer, H.: Extreme value laws in dynamical systems under physical observables, Physica D, 241, 497-513, 2012b.

Lai, Y.-C.: Extreme fluctuations of finite-time Lyapunov exponents in chaotic systems, Int. J. Modern Phys. B, 21, 3941-3946, 2007.

Leadbetter, M., Lindgren, G., and Rootzén, H.: Extremes and related properties of random sequences and processes, Springer Series in Statistics, Springer-Verlag, New York, 1983.

Leutbecher, M. and Palmer, T.: Ensemble forecasting, J. Comput. Phys., 227, 3515-3539, 2008.

Lorenz, E.: Deterministic nonperiodic flow, J. Atmos. Sci., 20, 130141, 1963.

Lorenz, E.: Predictability-A problem partly solved, in: Predictability of Weather and Climate, edited by: Palmer, T. and Hagedorn, R., Cambridge University Press, 40-58, 1996.

Mailier, P., Stephenson, D., Ferro, C., and Hodges, K.: Serial clustering of extratropical cyclones, Mon. Wea. Rev., 134, 22242240, 2006.

Nese, J.: Quantifying local predictability in phase space, Physica D, 35, 237-250, 1989.

Oseledec, V.: A multiplicative ergodic theorem: Lyapunov characteristic numbers for dynamical systems, Trudy Mosk. Mat. Obsc. 19, 197, 1968.

Palmer, T., Gelaro, R., Barkmeijer, J., and Buizza, R.: Singular vectors, metrics and adaptive observations, J. Atmos. Sci., 55, 633653, 1998.

Pomeau, Y. and Manneville, P.: Intermittent Transition to Turbulence in Dissipative Dynamical Systems, Commun. Math. Phys., 74, 189-197, 1980.

Prasad, A. and Ramaswamy, R.: Characteristic distributions of finite-time Lyapunov exponents, Phys. Rev. E, 60, 2761-2766, 1999.

Sterk, A., Vitolo, R., Broer, H., Simó, C., and Dijkstra, H.: New nonlinear mechanisms of midlatitude atmospheric low-frequency variability, Physica D, 239, 702-718, 2010.

Trevisan, A. and Palatella, L.: On the Kalman Filter error covariance collapse into the unstable subspace, Nonlin. Processes Geophys., 18, 243-250, doi:10.5194/npg-18-243-2011, 2011.

Vitolo, R., Stephenson, D., Cook, I., and Mitchell-Wallace, K.: Serial clustering of intense European storms, Meteorol. Z., 18, 411424, 2009.

Zaldívar, J.-M., Bosch, J., Strozzi, F., and Zbilut, J.: Early warning detection of runaway initiation using non-linear approaches, Commun. Nonlin. Sci. Numer. Simul., 10, 299-311, 2005.

Ziehmann, C., Smith, L., and Kurths, J.: The bootstrap and Lyapunov exponents in deterministic chaos, Physica D, 126, 49-59, 1999.

Ziehmann, C., Smith, L., and Kurths, J.: Localized Lyapunov exponents and the prediction of predictability, Phys. Lett. A, 271, 237-251, 2000. 